\title{
Tricritical spiral vortex instability in cross-slot flow
}

\author{
Simon J. Haward, ${ }^{1}$ Robert J. Poole, ${ }^{2}$ Manuel A. Alves, ${ }^{3}$ Paulo J. Oliveira, ${ }^{4}$ Nigel Goldenfeld,${ }^{5}$ and Amy Q. Shen ${ }^{1}$ \\ ${ }^{1}$ Okinawa Institute of Science and Technology Graduate University, Onna, Okinawa 904-0495, Japan \\ ${ }^{2}$ School of Engineering, University of Liverpool, Brownlow Street, Liverpool L69 3GH, United Kingdom \\ ${ }^{3}$ Faculdade de Engenharia da Universidade do Porto, DEQ, CEFT, Rua Dr. Roberto Frias, 4200-465 Porto, Portugal \\ ${ }^{4}$ Departamento de Engenharia Electromecânica, C-MAST, Universidade Beira Interior, 6201-001 Covilhã, Portugal \\ ${ }^{5}$ Department of Physics, University of Illinois at Urbana-Champaign, Loomis Laboratory of Physics, 1110 West Green Street, \\ Urbana, Illinois 61801-3080, USA
}

(Received 30 August 2015; revised manuscript received 9 November 2015; published 9 March 2016)

\begin{abstract}
We examine fluid flow through cross-slot devices with various depth to width ratios $\alpha$. At low Reynolds number, Re, flow is symmetric and a sharp boundary exists between the two incoming fluid streams. Above an $\alpha$-dependent critical value, $\operatorname{Re}_{c}(\alpha)$, a steady symmetry-breaking bifurcation occurs and a spiral vortex structure develops. Order parameters characterizing the instability grow according to a sixth-order Landau potential, and show a progression from second- to first-order transitions as $\alpha$ increases beyond a tricritical value of $\alpha \approx 0.55$. Flow simulations indicate the instability is driven by vortex stretching at the stagnation point.
\end{abstract}

DOI: 10.1103/PhysRevE.93.031101

Vortices are a ubiquitous hallmark of flow complexity and turbulence. But how do vortices form in a fluid bulk away from containing walls, in the absence of shear and before the onset of turbulence? We have examined the formation of vortices above a moderate critical Reynolds number during steady laminar flow in the prototypical cross-slot geometry. The change in flow pattern is as distinctive as the classical Taylor vortex formation [1], but we show that it is driven by the extensional flow near the stagnation point rather than by centrifugal effects.

The planar elongational flow field generated by the crossslot geometry [Fig. 1(a)] has applications in many areas of research, including studies of macromolecular dynamics and viscoelastic fluid properties [2-7], and for imposing controlled deformations to cells and vesicles [8-10]. It has been known since the early 1990s that such intersecting flows are prone to instability beyond a modest critical Reynolds number $\operatorname{Re}_{c} \sim$ $O(10-100)$. In very deep cross-slot channels the instability takes the form of a stack of three-dimensional vortical structures that appear in the central crossover region $[11,12]$. In the related four-roll mill apparatus [13] a single vortical region has been observed [14]. More recently, in microscale cross-slot devices, a flow instability of similar appearance has been shown to promote mechanical scission of polymer chains [15], and also to generate enhanced mixing at low Re $[16,17]$. However, to date this instability has still not been characterized in any significant detail. The cross-slot can be considered as a fundamental base-case geometry representative of planar intersecting and stagnation point forming flow geometries in general. Improved understanding and characterization of flow stability conditions in the cross slot is thus relevant to a wide range of fluidic systems.

Published by the American Physical Society under the terms of the Creative Commons Attribution 3.0 License. Further distribution of this work must maintain attribution to the author(s) and the published article's title, journal citation, and DOI.
In this work, we report the results of detailed experimental and numerical studies of the spiral vortex flow instability in cross slots with a range of aspect ratios (depth to width ratios $0.4 \leqslant \alpha \leqslant 3.87$ ) and over a range of Reynolds number $1 \lesssim \operatorname{Re} \lesssim 150$. We find that the instability occurs above an $\alpha$-dependent critical value $\operatorname{Re}_{c}$ and results in a single vortex that extends downstream along the outlet channels and remains steady in time [see Fig. 1(b)]. We identify order parameters that characterize the instability as a function of Re in both the experiments and the simulations and we present a systematic analysis in terms of bifurcation theory analogous to the Landau theory of equilibrium phase transitions. The observed phenomena are well described by a Landau-type sixth-order polynomial potential [19-21], with parameters that show the transition develops from second order to first order as $\alpha$ increases. A tricritical point occurs for $\alpha \approx 0.55$, and the universal scaling function and behavior are measured near the tricritical point. Time-resolved numerical simulations indicate that this is a new category of fluid mechanical instability, which is initiated by the formation of Dean vortices [22] and driven by vortex stretching at the stagnation point.

The cross-slot device [Fig. 1(a)] consists of bisecting rectangular channels of width $w$ and depth $d$, with aspect ratio $\alpha=d / w$. Four experimental devices are utilized, all with $d=1.2 \mathrm{~mm}$ but with $w$ varied in order to provide $\alpha=$ $0.49,1.00,1.85$, and 3.87. An inlet length $\geqslant 12.5 w$ ensures fully developed flow before fluid reaches the central region of each device. Water is pumped into two opposing channels (along the $y$ direction) and exits through the two opposing outlet channels ( $x$ direction). One of the incoming fluid streams is fluorescently dyed with rhodamine B (molar concentration $c=10 \mu \mathrm{M}$, Sigma Aldrich). A laser-scanning confocal microscope (Zeiss LSM 780) is employed to examine the interface between fluid streams in the central crossover region, enabling visualization of flow structures in the $x=0$ plane [(green) shaded area in Fig. 1(a)]. Experiments are performed at $24^{\circ} \mathrm{C}$ over a range of $\operatorname{Re}=\rho w U / \mu$, where the fluid density $\rho=$ $997.1 \mathrm{~kg} \mathrm{~m}^{-3}$ and the dynamic viscosity $\mu=9.1 \times 10^{-4}$ Pa s. 
(a)

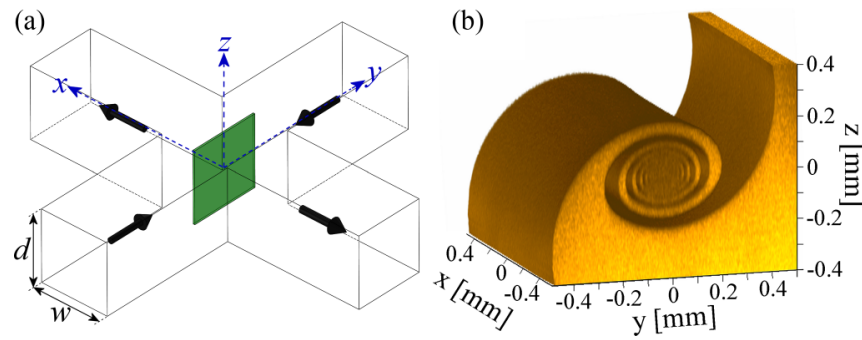

FIG. 1. (a) Schematic diagram of a cross-slot device. Flow enters along $y$ and exits along $x$. Confocal microscopy is performed in $z$ planes, which are scanned through the full depth of the device and used to reconstruct an image in the $x=0$ plane [shaded (green) region]. (b) 3D rendering of a vortex structure observed for the flow of water at $\operatorname{Re}=75.8$ in a cross slot with $\alpha=1$. The image is generated from $z$-plane images spaced at $\delta z=5 \mu \mathrm{m}$ and has been cropped around the central vortex. The volume shown corresponds to the fluorescently dyed fluid stream. Also see Movie M1 for an animated version of Fig. 1(b) [18].

The average flow velocity $U$ is controlled using a precision dual syringe pump (Harvard PHD Ultra). While for low Re the interface between fluid streams is sharp and vertical over the $y=0$ plane, beyond a fairly moderate critical value $\operatorname{Re}_{c}$ the flow bifurcates and breaks symmetry (though remains laminar and steady in time) and intricate three-dimensional (3D) spiral vortex structures develop [Fig. 1(b), Movie M1].

Due to the time required for experimental image aquisition ( $\sim 2$ min at each flow rate) performing quasistatic variations in Re through the transition is not possible. Therefore, to reveal the details of the critical Reynolds numbers and resolve hysteresis in the transition we perform complimentary numerical simulations. The numerical method solves the equations of motion and mass conservation for laminar flow of a Newtonian incompressible fluid using an implicit, second-order finite volume method $[5,23,24]$. Nonuniform orthogonal meshes with increasing degree of refinement are deployed on the 3D cross-slot geometry in order to ensure numerical accuracy. Theoretical fully developed velocity profiles are applied at the inlets, while at the outlets zero axial gradients are assumed and the total flow rate is forced to satisfy overall mass conservation. The numerical simulations explore devices with aspect ratios set equal to the four experimental devices as well as additional aspect ratios near to the tricritical value.

In Figs. 2(a)-2(d) we present confocal images of the $x=0$ plane for the cross-slot device with $\alpha=1$, showing the evolution of the vortical structure as Re increases. Qualitatively similar behavior is observed in all four geometries (see Movies M2-M5 [18]): at low Re the interface between fluid streams is sharp and vertical as expected; however, as $\mathrm{Re}$ increases above a critical value the spiral vortex forms abruptly about the $x$ axis. The experimental value of the critical Reynolds number $\operatorname{Re}_{c \text {,expt }}$ for the appearance of the spiral depends on the aspect ratio $\left(\operatorname{Re}_{c \text {,expt }} \approx 100,40,24\right.$, and 26 for $\alpha=0.49,1.00,1.85$, and 3.87, respectively). With further increases in $\mathrm{Re}$, the spatial extent of the spiral expands, as does the number of turns of the arms. The sharply defined images of the spiral vortex structure provide a clear visual indication of the steadiness of the flow at Reynolds numbers beyond the transition. In each of the four devices the central vortex has a favored orientation about the $x$ axis (anticlockwise for $\alpha=0.49$ and 1.00; clockwise for $\alpha=1.85$ and 3.87). The orientation is presumably biased by some minor geometrical imperfections in the devices, which cause the bifurcation to follow a favored branch.

Numerical simulations result in remarkably good agreement with the experiment, as shown by the streamline plots in Figs. 2(e)-2(h) that can be directly compared with the experimental results in Figs. 2(a)-2(d). Note that in the numerical simulations at $\alpha=1$ there is hysteresis in the transition and both symmetric and asymmetric steady solutions can be obtained for $\mathrm{Re}=42.8$ [Fig. 2(f)], depending on whether Re is quasistatically increased (decreased) from below (above) the transition (see Movie M6 [18]). The numerical simulations are particularly useful here because they reveal the presence of hysteresis on a scale that is too small for our experiments to resolve.

The experimental data is quantified by evaluating the standard deviation of the pixel intensity over images such as those shown in Figs. 2(a)-2(d) and Movies M2-M5 [18]. This provides the following bifurcation (or order) parameter:

$$
\psi_{\text {expt }}=1-2\left\langle(I-\langle I\rangle)^{2}\right\rangle^{1 / 2},
$$

where $I$ is the grayscale pixel value (normalized between 0 and 1) and $\langle\cdot\rangle$ indicates an average over all pixels in the field of view. This bifurcation parameter can be thought of as a kind of dilution-based mixing parameter [25,26]. For completely segregated fluid streams [similar to Fig. 2(a) in the prebifurcated state], there is a binary distribution of pixel intensities $(I=1$ or 0$)$, hence $\psi_{\text {expt }}=0$. Following the onset of the transition, intermediate pixel intensities are recorded within the vortex region, where diffusive mixing is enhanced by the increased interfacial area shared between fluid streams [Figs. 2(b)-2(d)]. Hence $\psi_{\text {expt }}$ becomes nonzero and increases as the vortex grows with increasing Re. Complete mixing between the fluid streams would mean a uniform pixel intensity of $I=0.5$ over the entire field of view, and hence $\psi_{\text {expt }}=1$. Figure 2(i) shows $\psi_{\text {expt }}$ as a function of $\operatorname{Re}$ for all four experimental aspect ratios.

In the numerical simulations, the instability is adequately described by the following order parameter:

$$
\psi_{\text {num }}=\frac{\left.v_{\max }\right|_{x=y=0}}{U},
$$

where $\left.v_{\max }\right|_{x=y=0}$ is the maximum value of the $y$ component of the velocity measured along the $z$ axis at each $\operatorname{Re}$ (for a symmetric flow $\left.\psi_{\text {num }} \equiv 0\right)$. Figure $2(\mathrm{j})$ shows $\psi_{\text {num }}$ as a function of the control parameter $\varepsilon=\left(\operatorname{Re}-\operatorname{Re}_{c}\right) / \operatorname{Re}_{c}$ for various aspect ratios. The data is fitted using a Landau-type model with "free energy" $F$, given by

$$
F=-h \psi-\frac{1}{2} \varepsilon \psi^{2}+\frac{g}{4} \psi^{4}+\frac{k}{6} \psi^{6},
$$

where we set the order parameter $\psi \equiv \psi_{\text {num. }}$. Even terms are included in Eq. (3) since for a perfect system $F$ should be independent of the sign of $\psi$ (i.e., the handedness of the spiral). The lowest order asymmetric "field" term $(h \psi)$ accounts for imperfections that bias the handedness. In the numerical simulations the vortex forms in the clockwise or anticlockwise 

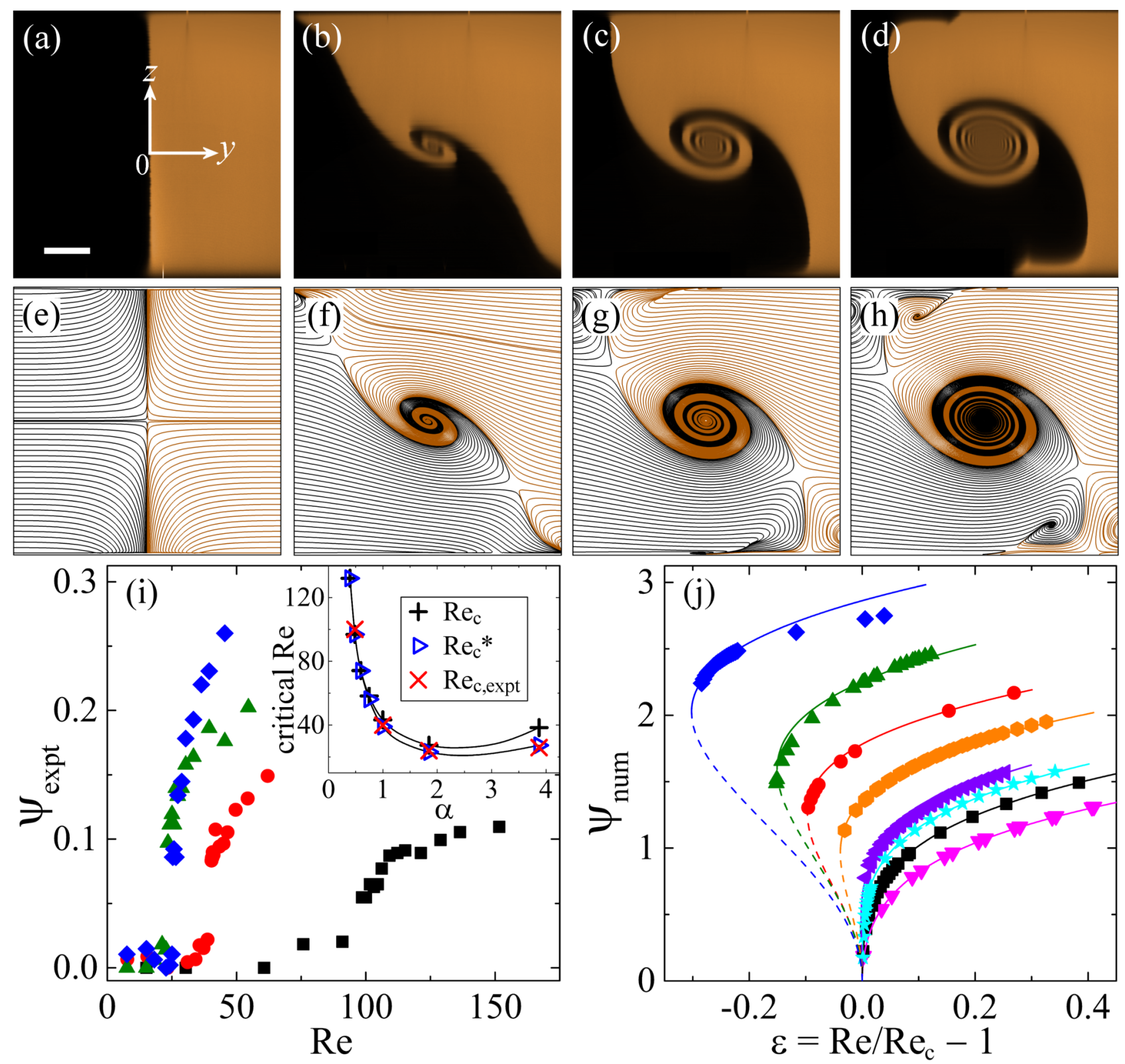

FIG. 2. Confocal imaging [(a)-(d)] and numerically generated streamlines [(e)-(h)] depicting the evolution of flow structures in the $x=0$ plane for Newtonian fluid flow in the cross-slot device with $\alpha=1$ under the following conditions: (a), (e) $\operatorname{Re}=15.2$; (b), (f) $\operatorname{Re}=42.8$; (c), (g) $\operatorname{Re}=60.6$; and (d), (h) $\operatorname{Re}=91.0$. In (a)-(d) fluorescently dyed fluid enters from the right (positive $y$ ) and undyed fluid enters from the left (negative $y$ ); outflow is along $x$ (i.e., normal to the page). Scale bar in (a) represents $200 \mu \mathrm{m}$. In (e)-(h) the streamlines are colored in order to resemble the experiment. The numerical result shown in (f) is one of two possible solutions at this Re; a symmetric solution can also be obtained (see Movie M6 [18]). (i) Bifurcation parameter in all four experimental cross-slot devices, evaluated according to Eq. (1). The inset shows experimental and numerical critical Reynolds numbers for the transition. (j) Numerical order parameter as a function of the control parameter, $\varepsilon$, fitted with a Landau sixth-order polynomial potential, Eqs. (3) and (4); broken lines represent unstable branches. Legend for parts (i) and (j): $\nabla, \alpha=0.4 ; \boldsymbol{\square}, \alpha=0.49 ; \star, \alpha=0.55 ; \triangleleft, \alpha=0.6 ;-\alpha=0.75 ; \boldsymbol{\bullet}, \alpha=1 ; \boldsymbol{\Delta}, \alpha=1.85 ; \diamond, \alpha=3.87$.

orientation with similar probability, hence $h=0$ (for all $\alpha$ ). For given values of the parameters $h, g$, and $k$, the value of $\psi(\varepsilon)$ corresponds to the extrema of $F$, i.e., $\partial F / \partial \psi=0$, giving

$$
\varepsilon \equiv \operatorname{Re} / \operatorname{Re}_{c}-1=k \psi^{4}+g \psi^{2}-h \psi^{-1} .
$$

For $\alpha \lesssim 0.55$, the ratio of the coefficients $g / k>0$, which means bifurcation is forward or supercritical, and corresponds to a second-order transition. With increasing $\alpha, g / k$ decreases monotonically. For $\alpha \gtrsim 0.55, g / k$ turns negative and the bifurcation becomes backwards or subcritical, corresponding to a first-order transition. An increasingly large hysteresis loop grows as $\alpha$ is increased further. The numerical data for $\alpha=0.55$ [(cyan) stars in Fig. 2(j)] are well fitted by Eq. (4) with $g=h=0$, (i.e., $\varepsilon=k \psi^{4}$ ) and thus corresponds to a tricritical transition. Fitting of the numerical data with Eq. (4) provides the values of $\operatorname{Re}_{c}$ shown in the inset to Fig. 2(i). For $\alpha \gtrsim 0.55$, due to the hysteresis loop we can find two values of the critical Reynolds number: $\operatorname{Re}_{c}\left(\operatorname{Re}_{c}^{*}\right)$ denotes the value 




FIG. 3. Collapse of experimental data (solid symbols) and numerical data (hollow symbols) onto the theoretical curve given by Eq. (5) (solid lines). The inset shows the experimental bifurcation parameter fitted with the sextic Landau potential suggested by the numerical results. $\nabla, \alpha=0.4 ; \square, \alpha=0.49$;,$\alpha=0.55 ; \triangleleft, \alpha=0.6$; $\diamond, \alpha=0.75 ; \bigcirc, \alpha=1 ; \triangle, \alpha=1.85 ; \diamond, \alpha=3.87$. Solid symbols are experimental and open symbols are numerical data points.

found for quasistatic increases (decreases) in Re from below (above) the onset. Our experimental critical Reynolds numbers $\left(\operatorname{Re}_{c \text {,expt }}\right)$ agree well (within approximately $\left.\pm 5 \%\right)$ with the numerical values of $\mathrm{Re}_{c}^{*}$.

The theory for tricritical points [27] predicts a universal scaling form for the canonical order parameter $\phi \equiv \psi \sqrt{k /|g|}$ in terms of the control parameter $r \equiv \varepsilon k / g^{2}$, showing data collapse (for $h=0$ ) in the form

$$
\phi^{2}=[-\operatorname{sgn}(g)+\sqrt{1+4 r}] / 2 .
$$

In Fig. 3, we plot both the experimental and numerical results in scaled form and show the comparison with the data collapse prediction. The agreement is excellent and confirms our identification of the spiral vortex transition as a tricritical one.

The inset to Fig. 3 shows the experimental bifurcation parameter fitted with Eq. (4). Here, $\operatorname{Re}_{c}$ is set to the value determined numerically for each aspect ratio and a small asymmetric coefficient $0.002<h<0.003$ is introduced to account for geometrical imperfections in the experimental devices. The experimental order parameter is well described by the same sextic Landau potential that describes the numerical data.

The tricritical point instability in the cross slot has a clearly distinct mechanism from that reported for flow in low-aspect ratio Taylor-Couette devices above a critical angular velocity (e.g., [20,21]). It is not a purely centrifugal instability, such as arises in Taylor-Couette [1] or Dean [22] flows (neither is it a shear instability, i.e., Kelvin-Helmholtz, or due to wall turbulence). Time-resolved numerical simulations indicate that it can be classified as a stagnation point instability coupled to vortex stretching. As $\mathrm{Re}$ is increased beyond $\approx 15$, centrifugal instability due to flow around the corners of the cross slot results in the formation of a symmetrical four-cell pattern of Dean vortices in the channel cross section (see Fig. 4 and Movie M7 [18]). This four-cell pattern renders
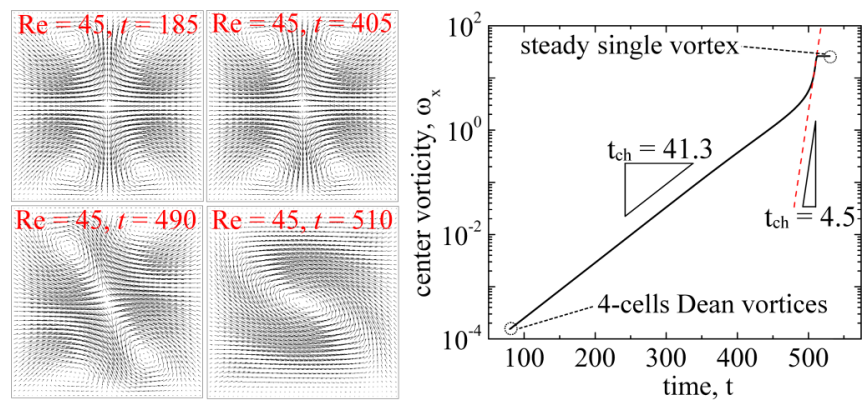

FIG. 4. Dynamic evolution of velocity vector fields and centerpoint vorticity following a step increase in the Re from 43 to 45 , computed from time-dependent numerical simulations for the case of $\alpha=1$. Also see Movie M7 [18] for a detailed time-resolved sequence of velocity fields.

the base flow susceptible to $y$-velocity disturbances along the $z$ axis. Small lateral displacements of fluid elements are amplified by the Dean cells and, by continuity, are antisymmetric about $z=0$. This gives rise to an axial vorticity component, $\omega_{x}$. The dimensionless vorticity at the central point is governed by the dynamical equation $\partial \omega_{x} / \partial t=\omega_{x} \partial u / \partial x+$ $\operatorname{Re}^{-1}\left[\partial^{2} \omega_{x} / \partial x^{2}+\partial^{2} \omega_{x} / \partial y^{2}+\alpha^{-2} \partial^{2} \omega_{x} / \partial z^{2}\right]$, where $t$ is the dimensionless time and $u$ is the $x$ component of the velocity. The first and second terms on the right refer to vortex stretching and viscous diffusion, respectively. For $\operatorname{Re}<\operatorname{Re}_{c}$ the diffusion term dominates and viscosity dampens the initial disturbance. However, for $\operatorname{Re}>\operatorname{Re}_{c}$, simulations indicate that $\left(\partial \omega_{x} / \partial t\right) / \omega_{x}>0$ and the disturbance will grow. Growth is initially slow while the diffusion term controls the dynamics. Here the dimensionless characteristic time $t_{\text {ch }}$ defining the rate of growth is proportional to $\operatorname{Re} /\left(\operatorname{Re}-\mathrm{Re}_{c}\right)$ in this slowly growing regime. Once $\left|\omega_{x}\right|$ has grown sufficiently, the vortex stretching term dominates and a fast growth is observed until steady state is approached. As Fig. 4 shows, this regime of rapid spiral vortex formation is well described by an exponential fit with $t_{\mathrm{ch}}=4.5$.

In summary, we have demonstrated that the steady spiral vortex instability observed for flow driven far from equilibrium above a critical Reynolds number in cross-slot devices is well described by a Landau model analogous to that used near equilibrium tricritical points. The identification of a tricritical point is important because it predicts the complicated sequences of transitions and crossovers that occur in the Re- $\alpha$ phase diagram. In particular, the fact that there are lines of both supercritical and subcritical bifurcations in the $\varepsilon-g$ phase plane, allows one to predict the onset and growth of the spiral vortex structure and the extent to which hysteresis will be present. The recognition that a tricritical structure underlies this phenomenon opens the way for derivation of this structure from first principles (i.e., from the Navier-Stokes equations). It is likely that flow bifurcations in related geometries $[17,26,28,29]$ are driven by the same mechanism and can be characterized using the same tricritical point framework presented here. This will be valuable for understanding flow stability conditions in, and optimizing the performance of, fluidic systems that incorporate intersections and internal stagnation points. 
S.J.H. and A.Q.S. gratefully acknowledge the support of the Okinawa Institute of Science and Technology Graduate University with subsidy funding from the Cabinet Office, Government of Japan. M.A.A. acknowledges finan- cial support from the European Research Council (Grant Agreement No. 307499). We are indebted to Professor Helmut Brand and Professor Gregory Falkovich for helpful discussions.
[1] G. I. Taylor, Philos. Trans. R. Soc., A 223, 289 (1923).

[2] T. T. Perkins, D. E. Smith, and S. Chu, Science 276, 2016 (1997).

[3] C. M. Schroeder, H. P. Babcock, E. Shaqfeh, and S. Chu, Science 301, 1515 (2003).

[4] P. E. Arratia, C. C. Thomas, J. Diorio, and J. P. Gollub, Phys. Rev. Lett. 96, 144502 (2006).

[5] R. J. Poole, M. A. Alves, and P. J. Oliveira, Phys. Rev. Lett. 99, 164503 (2007).

[6] S. J. Haward and G. H. McKinley, Phys. Fluids 25, 083104 (2013).

[7] S. J. Haward, M. S. N. Oliveira, M. A. Alves, and G. H. McKinley, Phys. Rev. Lett. 109, 128301 (2012).

[8] V. Kantsler, E. Segre, and V. Steinberg, Phys. Rev. Lett. 101, 048101 (2008).

[9] V. Kantsler and R. E. Goldstein, Phys. Rev. Lett. 108, 038103 (2012).

[10] D. R. Gossett, H. T. K. Tse, S. A. Lee, Y. Ying, A. G. Lindgren, O. O. Yang, J. Rao, A. T. Clark, and D. Di Carlo, Proc. Natl. Acad. Sci. USA 109, 7630 (2012).

[11] V. N. Kalashnikov and M. G. Tsiklauri, Fluid Dyn. 26, 161 (1991).

[12] V. N. Kalashnikov and M. G. Tsiklauri, J. Non-Newtonian Fluid Mech. 48, 215 (1993).

[13] G. I. Taylor, Proc. R. Soc. London, Ser. A 146, 501 (1934).

[14] R. R. Lagnado and L. G. Leal, Exp. Fluids 9, 25 (1990).

[15] S. A. Vanapalli, S. L. Ceccio, and M. J. Solomon, Proc. Natl. Acad. Sci. USA 103, 16660 (2006).

[16] N. Ait Mouheb, A. Montillet, C. Solliec, J. Havlika, P. Legentilhomme, J. Comiti, and J. Tihon, Microfluid. Nanofluid. 10, 1185 (2011).

[17] N. Ait Mouheb, D. Malsch, A. Montillet, C. Solliec, and T. Henkel, Chem. Eng. Sci. 68, 278 (2012).
[18] See Supplemental Material at http://link.aps.org/supplemental/ 10.1103/PhysRevE.93.031101 for the following information. Movie M1 shows a three-dimensional rendering of a spiral vortex; Movies M2-M5 show the development of the vortex with increasing $\mathrm{Re}$ in cross slots with various $\alpha$; Movie M6 shows hysteresis in the numerical streamlines determined for quasistatic increases and decreases in $\operatorname{Re}$ for the case $\alpha=1$; Movie M7 shows a time sequence of numerically determined velocity vector fields following a step increase in Re from 43 to 45 for the case $\alpha=1$.

[19] L. Landau, Phys. Z. Sowjetunion 11, 26 (1937), reprinted in Collected Papers of L. D. Landau, edited by D. ter Haar (Gordon and Breach, New York, 1965), p. 193.

[20] A. Aitta, G. Ahlers, and D. S. Cannell, Phys. Rev. Lett. 54, 673 (1985).

[21] A. Aitta, Phys. Rev. A 34, 2086 (1986).

[22] W. R. Dean, Philos. Mag. 5, 673 (1928).

[23] M. A. Alves, P. J. Oliveira, and F. T. Pinho, Int. J. Numer. Methods Fluids 41, 47 (2003).

[24] R. J. Poole, G. N. Rocha, and P. J. Oliveira, Comput. Fluids 93, 91 (2014).

[25] A. D. Stroock, S. K. W. Dertinger, A. Ajdari, I. Mezic, H. A. Stone, and G. M. Whitesides, Science 295, 647 (2002).

[26] J. Aubin, M. Ferrando, and V. Jiricny, Chem. Eng. Sci. 65, 2065 (2010).

[27] N. Goldenfeld, Lectures on Phase Transitions and the Renormalization Group (Addison-Wesley, Advanced Book Program, Reading, MA, 1992).

[28] S. Sarkar, K. K. Singh, V. Shankar, and K. T. Shenoy, Chem. Eng. Process. 85, 227 (2014).

[29] K. K. Chen, C. W. Rowley, and H. A. Stone, Phys. Fluids 27, 034107 (2015). 